\title{
MULTIPLIER REPRESENTATIONS OF DISCRETE GROUPS
}

\author{
ADAM KLEPPNER
}

\begin{abstract}
Let $\sigma$ be a multiplier on the discrete group $G$. Extending theorems of Kaniuth and Thoma to the case of multiplier representations, we determine when the left regular $\sigma$ representation of $G$ has a type I subrepresentation, and when all the $\sigma$ representations of $G$ are type I.
\end{abstract}

1. Let $\sigma$ be a normalized multiplier on the discrete group $G$ (cf. $[1,7,9]$ ). An element $x \in G$ is $\sigma$-regular if $\sigma(x, a)=\sigma(a, x)$ for all $a$ which commute with $x$. If $x$ is $\sigma$-regular so is every conjugate of $x$ [7, Lemma 3], and we may speak of the $\sigma$-regular conjugacy classes in $G$. Let $\Delta_{0}=\Delta_{0}(\sigma)$ be the set of elements lying in finite $\sigma$-regular conjugacy classes, let $\Delta=\Delta(\sigma)$ be the subgroup generated by $\Delta_{0}$, and let $\Delta^{\prime}$ be its commutator subgroup. The left regular $\sigma$ representation $\lambda_{\sigma}=\lambda_{G, \sigma}$ acts on $l^{2}(G)$ by

$$
\lambda_{\sigma}(x) f(y)=\sigma\left(x^{-1}, y\right) f\left(x^{-1} y\right), \quad f \in l^{2}(G), x, y \in G .
$$

It is a finite representation [7, Theorem 9]. We say that the multiplier $\sigma$ is type I if all the primary $\sigma$ representations are type $I$.

THEOREM 1. $\lambda_{\sigma}$ has a type I subrepresentation if and only if the following three conditions are satisfied:

(a) $[G: \Delta]<\infty$.

(b) $\left|\Delta^{\prime}\right|<\infty$.

(c) $\sigma$ is trivial on some cofinite subgroup.

THEOREM 2. The following are equivalent:

(a) $\lambda_{\sigma}$ is type I.

(b) $\sigma$ is type $\mathrm{I}$.

(c) $\sigma$ is trivial on some cofinite abelian subgroup.

For the case of ordinary representations $(\sigma=1)$, Theorem 1 is due to Kaniuth [6] and Theorem 2 to Thoma $[15,16]$. Smith [13] gave a global proof of these theorems, and the proof of the necessity of (a) and (b) in Theorem 1 is essentially her proof. Though it is quicker to use Thoma's theorem in the proof of Theorem 2, we indicate another method which gives an alternate proof of Thoma's theorem. Duflo [3] has also found a version of Theorem 2. After completing most of this work, I learned that A. K. Holzherr [4] had also found, by somewhat different methods, Theorem 2,

Received by the editors February 8, 1982 and, in revised form, November 15, 1982.

1980 Mathematics Subject Classification. Primary 22D10; Secondary 20C25. 
most of Theorem 1 and a number of other related results. In particular, he identified the projection on the type I part of $\lambda_{\sigma}$. I am indebted to the referee for pointing out the correct formulation of Theorem 1 .

2. For each subset $S \subset G$ we denote by $Z(S)=Z_{G}(S)$ its centralizer in $G$ and we put

$$
Z_{\sigma}(S)=\{y \in Z(S) \mid \sigma(x, y)=\sigma(y, x), \text { all } x \in S\} .
$$

If $S=\{x\}$ we write shortly $Z(x)$ or $Z_{\sigma}(z) . x$ is $\sigma$ regular if and only if $Z_{\sigma}(x)=Z(x)$. Note that $x \in Z_{\sigma} \Leftrightarrow y \in Z_{\sigma}(x)$ and $Z_{\sigma}(S)=\bigcap_{x \in S} Z_{\sigma}(x)$.

LEMma 1. (a) For each subset $S \subset G, Z_{\sigma}(S)$ is a subgroup.

(b) If $H$ is a finitely generated subgroup of $\Delta$ then $\left[G: Z_{\sigma}(H)\right]<\infty$.

Proof. Osima [11] observed that $y \rightarrow \sigma(x, y) \sigma(y, x)^{-1}, y \in Z(x)$, is a character of $Z(x) . Z_{\sigma}(x)$ is the kernel of this character. Thus $Z_{\sigma}(x)$ and $Z_{\sigma}(S)$ are subgroups. It is easy to check that $Z_{\sigma}(a) \cap Z_{\sigma}(b) \subset Z(a b)$. If $a \in \Delta_{0}, Z_{\sigma}(a)=Z(a)$ is cofinite. If $x=a_{1} a_{2} \cdots a_{n} \in \Delta$, then $\bigcap_{1}^{n} Z_{\sigma}\left(a_{i}\right) \subset Z_{\sigma}(x)$. Thus $Z_{\sigma}(x)$ is cofinite. If $H$ is generated by $\left\{x_{1}, \ldots, x_{m}\right\} \subset \Delta$ and $y \in H$, then $\cap Z_{\sigma}\left(x_{i}\right) \subset Z_{\sigma}(y)$, and $\cap Z_{\sigma}\left(x_{i}\right) \subset$ $\bigcap_{y \in H} Z_{\sigma}(y)=Z_{\sigma}(H)$ is also cofinite.

3. To prove that (a), (b), (c) in Theorem 1 are necessary, we begin by observing that if the set $\Delta_{0}$ is a subgroup of infinite index, then by [7, Theorem 4] $\lambda_{\sigma}$ is type II. However, the proof of that theorem is valid also in the case that the subgroup $\Delta$ generated by $\Delta_{0}$ is of infinite index. Thus in what follows we shall assume $\Delta$ is cofinite. Let $\mathscr{Q}(G)$ be the bicommutant of $\lambda_{\sigma}(G)$, and if $E$ is a projection in $\mathscr{Q}(G)^{\prime}$, let $\mathcal{U}_{E}(G)$ be the corresponding reduced algebra.

LEMMA 2 (SMITH [13]). Let $G$ be an FC group, $H$ a subgroup, $E$ a central projection in $\mathscr{Q}(G)$ so that $\mathscr{U}_{E}(G)$ and $\mathscr{U}_{E}(H)$ are type $\mathrm{I}_{n}$, for some $n<\infty$. Then $\left[Z_{\sigma}(H), G\right]$ is finite.

Smith's proof of this lemma [13, Lemma 2] for the case $\sigma=1$ may be adopted, after replacing the group algebra by the appropriate twisted group algebra, almost without change to the present setting.

Observing that $\Delta$ is contained in the subgroup of elements lying in finite conjugacy classes, we see that the remainder of Smith's proof for the case $\sigma=1$ [13, pp. 403-404] that if $\lambda_{\sigma}$ has a type I summand, then $\left|\Delta^{\prime}\right|<\infty$, is valid for arbitrary $\sigma$. This proves the necessity of (a) and (b) in Theorem 1 .

To show there is a cofinite subgroup of $G$ on which $\sigma$ is trivial, it is enough to show there is a cofinite subgroup of $\Delta$ on which $\sigma$ is trivial. Thus we may suppose $G=\Delta$ and $[G, G]=G^{\prime}$ is finite. Put $H=Z_{\sigma}\left(G^{\prime}\right)$ and $F=G^{\prime} \cap H$. By Lemma $1, H$ is cofinite. $F$ is finite and $[H, H] \subset F \subset \operatorname{center}(H)$.

Lemma 3. $\left.\sigma\right|_{H \times H}$ is similar to a multiplier lifted from a multiplier $\sigma^{\prime}$ on $H / F$.

Proof. If $\gamma$ is an irreducible $\sigma$ representation of $H$, then for all $a \in F$ and $x \in H$,

$$
\gamma(a) \gamma(x)=\sigma(a, x) \gamma(a x)=\sigma(x, a) \gamma(x a)=\gamma(x) \gamma(a)
$$


Hence $\gamma(a)$ is a multiple $\varphi(a)$ of the identity, i.e. $\gamma(a)=\varphi(a) \cdot 1$, where $|\varphi(a)|=1$. Thus $F \subset$ projective kernel of $\gamma[2, \S 1]$, and $\left.\sigma\right|_{H \times H}$ is similar to a multiplier lifted from a multiplier $\sigma^{\prime}$ on $H / F$ [2, Lemma 1.3].

Replacing $\left.\sigma\right|_{H \times H}$ by that multiplier, we may suppose $\sigma(x, y)=\sigma(\bar{x}, \bar{y})$, all $x, y \in H$, where $\bar{x}=x F$.

Because $\lambda_{\sigma}$ is finite and has a type I part, it contains a finite-dimensional subrepresentation, all of whose matrix coefficients vanish at $\infty$. The restriction of this representation to $H$ contains an irreducible subrepresentation $\pi$, all of whose matrix coefficients vanish at $\infty$. If $n=\operatorname{dim} \pi$, then $\sigma^{n}(x, y)=$ det $\pi(x)$ det $\pi(y)$ det $\pi(x y)^{-1}$. Thus we may replace $\sigma$ by a similar multiplier and assume $\sigma^{n}=1$; that is, we may assume $\sigma$ takes its values in a finite subgroup $C$ of the circle.

Consider now the extension $1 \rightarrow C \rightarrow H^{\sigma} \rightarrow H \rightarrow 1$ of $C$ by $H$ defined by $\sigma$. Put $\pi^{0}(t, x)=t \pi(x), x \in H, t \in C$. Then $\pi^{0}$ is a representation of the discrete group $H^{\sigma}$, all of whose matrix coefficients vanish at $\infty$. By a theorem of Taylor [14, Theorem 1, Corollary 1], there is a finite normal subgroup $K$ of $H^{\sigma}$ such that $H^{\sigma} / K$ is a Moore group (only finite-dimensional unitary representations) and $K \subset \operatorname{ker} \pi^{0}$, i.e., $\pi^{0}$ is lifted from a representation of $H^{\sigma} / K . H^{\sigma} / K$ is, in particular, type I, and by Thoma's theorem $[13,15,16]$ there is a cofinite subgroup $M \subset H^{\sigma}$ so that $M / K$ is abelian. Then $P=M / K \cap(H / H \cap K)$ is a cofinite abelian subgroup of $H / H \cap K$ $\subset H^{\sigma} / K$. Since $K \subset \operatorname{ker} \pi^{0}, K \cap H \subset \operatorname{ker} \pi \subset$ projective kernel of $\pi$. Thus $\left.\sigma\right|_{H \times H}$ is similar to a multiplier lifted from a multiplier $\sigma^{\prime \prime}$ of $H / H \cap K$ [2, Lemma 1.3]. Again replacing $\sigma$ by a similar multiplier, we may think of $\pi$ as a $\sigma^{\prime \prime}$ representation of $H / H \cap K$. Then $\left.\pi\right|_{P}$ is a finite-dimensional $\sigma^{\prime \prime}$ representation of the abelian group $P$. Hence there is a cofinite subgroup $Q \subset M, H \cap K \subset Q$, so that $\sigma^{\prime \prime}$ is trivial on $Q / H \cap K\left[2\right.$, Lemma 2.1, Theorem 3.1]. Then $\left.\sigma\right|_{Q \times Q}$ is trivial. We have the inclusion $Q \subset M \subset H \subset G$, and since each subgroup is of finite index in the next, $Q$ is cofinite in $G$.

This completes the proof of the necessity of the conditions in Theorem 1. To prove that they are sufficient we begin with

LEMma 4. Let $H$ be a subgroup of finite index in $G$ and $\pi$ a finite type I $\sigma$-representation of $H$. Then $\sigma$-Ind ${ }_{H}^{G} \pi$ is type $\mathrm{I}$.

(For the definition of $\boldsymbol{\sigma}$-induction see [ 2 or 9 ].)

Proof. $H$ contains a cofinite normal subgroup $N$ and $\left.\pi\right|_{N}$ is a finite type I representation. By the Mackey machine, $\sigma-\left.\operatorname{Ind}_{N}^{G} \pi\right|_{N}$ is also a finite type I representation, and it is easy to see that $\sigma$-Ind ${ }_{H}^{G} \pi \subset \sigma-\left.\operatorname{Ind}_{N}^{G} \pi\right|_{N}$.

Let $Q$ be a cofinite subgroup of $G$ on which $\sigma$ is trivial. Then $\Delta \cap Q^{\prime}=\Delta_{Q}$ is cofinite, $\left|\Delta_{Q}^{\prime}\right|<\infty$, and because $\Delta$ is contained in the subgroup of $G$ of elements in finite conjugacy classes, each $x \in \Delta_{Q}$ lies in a finite conjugacy class. By Kaniuth's theorem $\lambda_{\Delta_{Q}}$ (the left regular representation of $\Delta_{Q}$ ) has a type I part. By Lemma 4, $\lambda_{0}=\sigma$-Ind $\Delta_{\Delta_{Q}}^{G} \lambda_{\sigma}$ has a type I part. This completes the proof of Theorem 1.

This theorem can be presented in slightly different terms. For any group $H$ let $H_{F C}$ be the subgroup of elements lying in a finite conjugacy class. Note that $\Delta \subset G_{F C}$. 
THEOREM 1'. $\lambda_{\sigma}$ has a type I part if and only if the following three conditions are satisfied:

$\left(\mathrm{a}^{\prime}\right)\left[G: G_{F C}\right]<\infty$.

$\left(\mathrm{b}^{\prime}\right)\left|G_{F C}^{\prime}\right|<\infty$.

$\left(\mathrm{c}^{\prime}\right) \sigma$ is trivial on some cofinite subgroup.

In fact, suppose $\lambda_{\sigma}$ has type I part. Then $[G: \Delta]<\infty$ and because $\Delta \subset G_{F C}$, [G: $\left.G_{F C}\right]<\infty$. Since $\left|\Delta^{\prime}\right|<\infty$ and $\left[G_{F C}: \Delta\right]<\infty,\left|G_{F C}^{\prime}\right|<\infty$ [10, Lemma 4.1], and by Theorem 1, (c) is satisfied. Conversely, if $\sigma$ is trivial on a cofinite subgroup $Q$, then $Q_{F C}=Q \cap G_{F C}$ is cofinite in $Q$, and $Q_{F C}^{\prime} \subset G_{F C}^{\prime}$ is also finite. By Kaniuth's theorem $[6,13], \lambda_{Q}$ has a type I part, and by Lemma $4, \lambda_{\sigma}$ has a type I part.

4. The implication (c) $\Rightarrow(b)$ of Theorem 2 is a consequence of the Mackey machine [2,9]. In fact, if $H$ is a cofinite normal subgroup for which $\left.\sigma\right|_{H \times H}$ is type I, e.g., an abelian subgroup on which $\sigma$ is trivial, it follows from the Mackey machine that $\sigma$ is type $I$, and every cofinite subgroup contains a cofinite normal subgroup. (b) $\Rightarrow$ (a) is clear (see [12] for the case of uncountable groups). Thus what remains to show is $(a) \Rightarrow(c)$.

LEMMA 5. If $\lambda_{\sigma, G}$ is type I so is $\lambda_{\sigma, H}$ for every subgroup $H$.

This lemma is, in fact, valid for open subgroups of a locally compact group and is what is proved by Kallman in the proof of Proposition 2.4 of [5].

To complete the proof of Theorem 2 we need only observe that if $Q$ is a cofinite subgroup of $G$ on which $\sigma$ is trivial, then $\lambda_{\sigma, Q}=\lambda_{Q}$ is type I by Lemma 5 , and by Thoma's theorem $[13,15,16] Q$ contains a cofinite abelian subgroup.

5. It is possible to show (a) $\Rightarrow$ (c) in Theorem 2 without use of Thoma's theorem and obtain, in particular, another proof of that theorem. Assume $\lambda_{\sigma}$ is type I. Then by Theorem $1, \Delta$ is cofinite and $\Delta^{\prime}$ is finite. (This part of Theorem 1 did not use Thoma's theorem.) It is enough to show $\Delta$ contains a cofinite abelian subgroup on which $\sigma$ is trivial. Thus we may suppose that $G=\Delta$ and $G^{\prime}$ is finite. As in $\S 2$ we put $H=Z_{\sigma}\left(G^{\prime}\right), F=H \cap G^{\prime}$ and assume that $\sigma(x, y)=\sigma^{\prime}(\bar{x}, \bar{y}), x, y \in H$, where $\bar{x}=x F$. Let $p: H \rightarrow H / F$ be the canonical map.

We are assuming that $\lambda_{\sigma, G}$ is type I. By Lemma $5, \lambda_{\sigma, H}$ is type I. $\lambda_{\sigma^{\prime}, H / F} \circ p$ is a subrepresentation of $\lambda_{\sigma, H}$ (the map $f \rightarrow|F|^{-1} f \circ p$ is the intertwining operator). Thus $\lambda_{\sigma^{\prime}, H / F}$ is also type I. But $H / F$ is abelian and we know [2, Theorem 3.1, Lemma 3.1] that $\lambda_{\sigma^{\prime}, H / F}$ is type $\mathrm{I}$ if and only if there is a cofinite subgroup $K \subset H$ so that $\left.\sigma^{\prime}\right|_{K / F, K / F}$ is trivial, or what is the same, $\left.\sigma\right|_{K \times K}$ is trivial. Replacing $\sigma$ by a similar multiplier, we may suppose $\left.\sigma\right|_{K \times K}=1$. Then $\lambda_{\sigma, K}=\lambda_{K}$ is also type I (Lemma 5).

We now have an extension $1 \rightarrow F \rightarrow K \rightarrow P \rightarrow 1$, where $F$ is finite and central in $K, P$ is abelian, and $K$ has a type I regular representation. We apply the Mackey machine to describe $\lambda_{K}$. We have

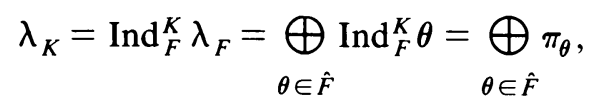

where $\pi_{\theta}=\operatorname{Ind}_{F}^{K} \theta$. Thus $\lambda_{K}$ is type $I$ if and only if each $\pi_{\theta}$ is type I. Since $K$ centralizes $F$, each $\theta \in \hat{F}$ may be extended to a projective character $\theta^{\prime}$ with Mackey 
obstruction $\omega_{\theta}^{-1}$, and $\pi_{\theta}=\theta^{\prime} \otimes \rho_{\theta}^{\prime \prime}$, where $\rho_{\theta}$ is the right regular $\omega_{\theta}$ representation of $P$ (here we think of $\omega_{\theta}$ as a multiplier on $P$ ), and $\rho_{\theta}^{\prime \prime}$ is its lift to $K$ (see [1]). Thus each $\pi_{\theta}$ is type $I$ if and only if each $\rho_{\theta}$ is type $I$, and this happens if and only if $\omega_{\theta}$ is trivial on a cofinite subgroup $Q_{\theta} \subset P$ [2, Theorem 3.1, Lemma 3.1]. Let $C$ be the inverse image in $K$ of $\bigcap_{\theta \in \hat{F}} Q_{\theta}$. $C$ is cofinite. We shall show it is abelian. Let $\pi \in \hat{C}$. Since $F$ is central in $C, \pi(a)=\theta_{\pi}(a) \cdot 1$, where $\theta_{\pi} \in \hat{F}, a \in F$. Then $x \rightarrow \theta_{\pi}^{\prime-1}(x) \pi(x)$ is constant on the $F$ cosets in $C$ and defines a representation $\pi^{*}$ of $C / F \subset P$ with multiplier $\omega_{\theta}$. Since $\omega_{\theta}$ is trivial on $C / F, \omega_{\theta}(x, y)=\omega_{\theta}(y, x)$, all $x, y \in C$ and $\pi^{*}(x) \pi^{*}(y)=\pi^{*}(y) \pi^{*}(x)$, all $x, y \in C / F$. Since $\pi^{*}$ is also irreducible, $\operatorname{dim} \pi^{*}=1$ $=\operatorname{dim} \pi$. Thus all irreducible representations of $C$ are 1-dimensional and $C$ is abelian.

\section{REFERENCES}

1. L. Baggett, $A$ weak containment theorem for groups with a quotient $R$-group, Trans. Amer. Math. Soc. 128 (1967), 277-290.

2. L. Baggett and A. Kleppner, Multiplier representations of abelian groups, J. Funct. Anal. 14 (1973), 299-324.

3. M. Duflo, Proc. Neptun Conference, Romania, 1980.

4. A. K. Holzherr, Discrete groups whose multiplier representations are type I, J. Austral. Math. Soc. Ser. A 31 (1981), 486-495.

5. R. Kallman, Certain topological groups are type I. II, Adv. in Math. 10 (1973), 221-255.

6. E. Kaniuth, Der typ der regulären Darstellungen diskreter Gruppen, Math. Ann. 182 (1969), 334-339.

7. A. Kleppner, The structure of some induced representations, Duke Math. J. 29 (1962), 555-572.

8. Multipliers on abelian groups, Math. Ann. 158 (1965), 11-34.

9. G. W. Mackey, Unitary representations of group extensions. I, Acta Math. 99 (1958), 265-311.

10. B. H. Neumann, Groups with finite classes of conjugate elements, Math. Z. 63 (1955), 76-96.

11. M. Osima, On the representations of groups of finite order, Math. J. Okayama Univ. 1 (1951), 33-61.

12. S. Sakai, On a characterization of type I $C^{*}$ algebras, Functional Analysis (Proc. Conf., Irvine, Calif., 1966), Thompson, Washington, D.C., 1967, pp. 138-143.

13. M. Smith, Regular representations of discrete groups, J. Funct. Anal. 11 (1972), 401-406.

14. K. Taylor, Group representations which vanish at infinity, Math. Ann. 251 (1980), 185-190.

15. E. Thoma, Über unitäre Darstellungen abzählbar, diskreter Gruppen, Math. Ann. 153 (1964), 111-138.

16. __ Eine characterisierung diskreter Gruppen vom typ I, Invent. Math. 6 (1968), 190-196.

Department of Mathematics, University of Maryland, College Park, Maryland 20742 